\title{
Neue Anthiciden und Mitteilungen über die Verbreitung bekannter Anthiciden.
}

Von Hans von Krekich-Strassoldo, Wien.

II. Teil.

(Mit 8 Figuren im Text.)

\section{Formicomus Andrewesi n. sp.}

Schlank, glänzend, Kopf und Halsschild tief rot, die Flügeldecken schwarz mit grünem Schimmer (manchmal ist die Basis der Flügeldecken etwas gerötet); Schenkel dunkelbraungelb, an der Basis (und am Vorderschenkeldorne des $\sigma^{\top}$ ) lichter; ebenso die Tarsen. Alle Tibien dunkel, nur an der Wurzel etwas rötlich; Fühler und Palpen rötlichgelb; die Endglieder der Fühler etwas gedunkelt.

Kopf kreisrund, die Rundung nur durch die hervorstehenden Augen unterbrochen; am Diskus fast glatt, an den Seiten und nach vorn etwas deutlicher, nicht grob und nicht tief punktiert; mit halb aufrechten, nach rückwärts gerichteten, etwas gekrümmten, dunklen, kurzen, dazwischen mit wenigen aufrechten längeren, gelblichen Haaren bekleidet. Fühler die Schultern erreichend; zweites Glied kürzer als das dritte, Endglied so lang wie das zehnte, spitz.

Halsschild von der Breite des Kopfes, $1 \frac{1}{3}$ mal länger als dieser,

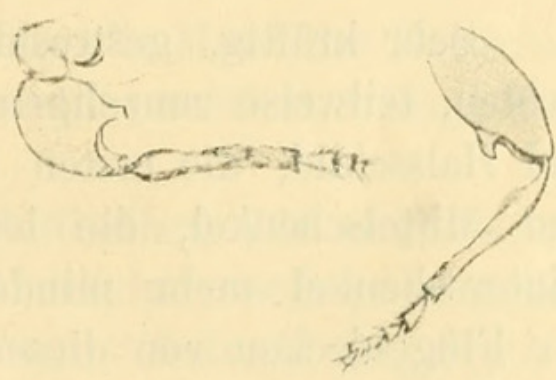

Fig. 1.

Fig. 2. an den Seiten allmälig erbreitert, dann nahe der Basis mäßig eingeschnürt und seitlich kräftig eingedrückt; stark und dicht punktiert; doch sind die seitlichen Ausbuchtungen des Halsschildes und eine halbmondförmige Stelle vor dem Basalrande fast ganz glatt. Behaarung wie am Kopfe.

Flügeldecken $2{ }^{1} / 2$ mal länger als in der Mitte breit, an der Basis $1 \frac{1}{2}$ mal so breit als der Halsschild, gegen die Mitte stark erbreitet, dann allmälig abnehmend und ziemlich spitz endend, Schultern etwas abfallend, aber mit deutlichen Ecken. Sehr seicht und zerstreut punktiert; aus jedem Punkte ein ziemlich langes, halb aufrechtes, gelbes Haar entspringend; dazwischen vereinzelte aufrecht stehende 
Haare. Seitenrand der Flügeldecken deutlich und vollständig, bis fast zum Nahtende reichend; hier ist der Rand beim $\sigma^{7}$ etwas aufgebogen. Schildchen klein, spitz, rot. Postskutellareindruck schwach. Nahtstreifen nur im letzten Viertel etwas erhoben.

Schenkel sehr keulig; Tibien und Tarsen kräftig. Die Vordertibien des $\sigma^{\pi}$ gleich nach der schmalen Wurzel stark verdickt und seitlich büschelförmig behaart, gegen die Spitze unten mit einer Anschwellung, die gleichfalls dicht behaart ist, versehen (Fig. 1). Vorderschenkel des 3 mit einem kräftigen, etwas abgestumpftem Dorne (Fig. 2).

L $0 \mathrm{ng}$. $5 \cdot 2 \mathrm{~mm}$.

Nilgiri Hills (Ostindien) in einer Höhe von $1000 \mathrm{~m}$ und darüber. Mai.

Dem Formic. rufopiceus Fairm. aus Belgaum (Ostindien) nahe stehend, aber von diesem durch die konstante, viel größere Gestalt, durch die Bildung der Vordertibien des $\sigma^{\top}$, durch die Punktierung des Kopfes (bei rofopiceus ist die Punktierung dicht und namentlich nach vorne gerunzelt) etc. verschieden.

Dem Herrn H. L. A ndrew es, der diesen Formicomus sammelte, zu Ehren benannt.

\section{Formicomus grossipes n. sp.}

Sehr kräftig, gestreckt, Kopf und Halsschild infolge der sehr dichten, teilweise runzeligen Punktierung fast matt erscheinend. Kopf und Halsschild, die ersten zwei bis drei Fühlerglieder, die Vorderund Mittelschenkel, die letzten Tarsenglieder und die Basis der Hinterschenkel mehr minder tief blutrot, manchmal auch die Basis der Flügeldecken von dieser Farbe. Fühler, Tibien, Hinterschenkel, die Tarsen zum Teile, endlich die Flügeldecken schwarz, letztere mit metallischem Schimmer.

Kopf elliptisch, hinten bogig, ganz wenig an der Basis abgestutzt; Augen sehr klein, wenig vortretend. Der Kopf ist mit kurzen anliegenden Haaren mäßig bekleidet. Fühler die Schultern erreichend, mit kräftigen, ziemlich geschwollenen, gegen die Spitze allmälig verdickten Gliedern; zweites Glied kürzer als das dritte; Endglied kaum länger als das zehnte, spitz. Palpen beilförmig, dunkel.

Halsschild sehr konvex, breiter als der Kopf, an den Seiten gegen die Mitte gleichmäßig, breit gerundet, oben vor dem deutlichen, glatten, nach vorwärts etwas ausgebuchteten Basalrande ziemlich plötzlich abfallend und zwei wenig deutliche Erhebungen bildend; die Seiten nahe dem Basalrande kräftig eingeschnürt. Sehr dicht, 
und in der Einschnürung runzelig punktiert; mit anliegenden feinen, kurzen, gegen die Einschnürung dichteren, gelblichen und mit einigen wenigen abstehenden Haaren bekleidet.

Flügeldecken an der Basis doppelt so breit als die Basis des Halsschildes, $21 / 2$ mal so lang als breit; gegen die Mitte allmälig, mäßig erbreitet, gegen die Spitze sehr verengt; Enden der Flügeldecken gemeinsam gerundet; mit sehr schwachem, postbasalem Eindrucke. Schultern deutlich, aber gerundet. Schildchen länglich, spitz. Nahtstreifen fein, in der zweiten Hälfte schwach erhoben. Punktierung ziemlich gleichmäßig, wenig dicht, nicht tief. Die ganze Oberseite mit feinen gelblichen, nicht ganz anliegenden Haaren ziemlich dicht bekleidet. Außerdem einige wenige abstehende Haare.

Schenkel außerordentlich kräftig und keulig. Vorderschenkel des or der Mitte, nach unten, mit einem scharfkantigen, spitz endenden Fortsatze(Fig. 3). Alle Tibien sehr kräftig, etwas gebogen, dunkel ; die Vordertibien des $\sigma^{7}$ unten gegen die Spitze erbreitert und kantig abgesetzt; Tarsen dunkel, nur die Endglieder etwas lichter; namentlich die Tarsen der Vorderfüße des o' mit zahl-

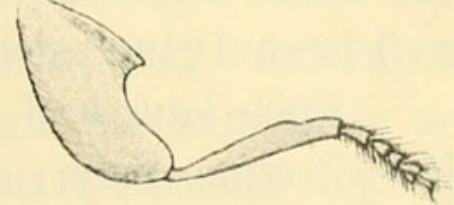

Fig. 3. reichen, zum Teile langen, dornartigen, sowie mit bürstenförmigen Haaren dicht besetzt.

Unterseite dunkelrot, nur die Kehle, die Mitte des Halsschildes und die Sternite schwarz; schwach und zerstreut punktiert, mäßig behaart. L ong. $4 \mathrm{~mm}$.

Nilgiri Hills (Tepu-Kader in Ostindien) $760 \mathrm{~m}$ Höhe. Mai.

Dem Formic. semiopacus Pic aus Pondichery sehr nahe stehend, aber durch den Mangel einer lichten, roten Schulterbinde, durch die kräftigere Gestalt, die stärkeren Fühler und Füße, durch etwas weniger konvexen Halsschild etc. verschieden.

\section{Formicomus Vethi n. sp.}

Körper schwarz, Flügeldecken mit bläulichem Schimmer, Füße, Fühler und Mundteile braunschwarz, sehr glänzend.

Kopf so lang als breit mit großen, nach rückwärts spitz auslaufenden, sehr vorstehenden, fein gerandeten Augen; nach rückwärts stark gerundet und in den nicht langen Hals auslaufend; mit zwei etwas schräg gestellten Eindrücken am Scheitel; vom Oberrande der Augen eine die Fühlerwurzel tangierende und vorne gerade sich vereinigende, schmale, erhobene Leiste; sehr schwach und zerstreut punktiert und mit zerstreuten, gelblichen Haaren bekleidet. 
Fühler lang, die Schultern erreichend, gegen die Spitze sehr mäßig verdickt, erstes Glied lang und verdickt, zweites Glied kürzer als das dritte, dieses kürzer als das vierte, die folgenden gleichmäßig lang, nur das Endglied etwas länger, spitz.

Halsschild länger und schmäler als der Kopf, fast doppelt so lang als breit, sehr konvex, vorne mäßig gerundet, mit ziemlich tiefem Seiteneindrucke, wie der Kopf punktiert und behaart; Basalrand deutlich.

Flügeldecken etwas länger als Kopf und Halsschild zusammen, an der Basis doppelt so breit als der Halsschild, an den Seiten mäßig erbreitert, an der Spitze einzeln gerundet; sehr schwach und sehr zerstreut punktiert, fast glatt, mit wenigen, zum Teile abstehenden gelblich-weißen Haaren bekleidet. Schultern etwas quer, mit spitzer Ecke; Schildchen länglich spitz, an seinen Seiten etwas erhoben und dann eingedruickt; kein Postskutellareindruck; Nahtstreifen nur im letzten Drittel sichtbar und etwas erhoben.

Füße lang und schlank, Schenkel sehr keulig; Vorderschenkel des $\sigma^{\pi}$ ohne Zahn; Vordertibien des $\sigma^{2}$ in der Mitte der Innenseite mit kräftiger, zahnartiger Ausbuchtung.

L ong. $4.5-5 \mathrm{~mm}$.

Preang er (Java).

Nahe bei $F$. sumatrensis Pic, aber durch den Mangel eines Zahnes oder eines Fortsatzes an der Innenseite der Vordertibien des 万ै sehr ausgezeichnet.

Herrn Dr. H. J. Veht in Haag, der mir diesen interessanten Formicomus zur Verfügung stellte, gewidmet. (Sammlung Veth und Hofmuseum in Wien.)

\section{Formicomus singularis n. sp.}

Ganz schwarz, Flügeldecken mit grünlichem Schimmer; groß, sehr glänzend.

Kopf fast so breit als lang; gleich hinter den Augen gerundet; diese groß, rund, stark vortretend; Scheitel ziemlich flach, an der

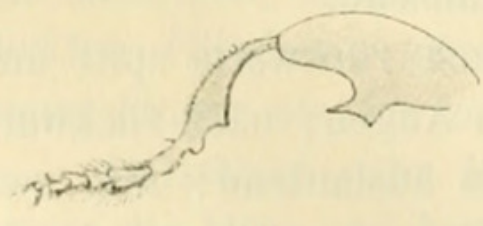

Fig. 4.

Fühlerwurzel etwas erhoben; am Augenrande und an der Stirne stärker und dichter, sonst schwach und zerstreut punktiert; mäßig, am Hinterkopfe etwas dichter (bürstenförmig, nach aufwärts) behaart. Fühler lang, schlank, die Mitte des Leibes erreichend; erstes Glied groß, stark verdickt, zweites Glied sehr kurz, drittes und viertes Glied ungefähr gleich lang, kürzer als das 
fünfte bis zehnte Glied, die ziemlich von gleicher Länge sind; gegen die Spitze mäßig verdickt, Endglied länger als das zehnte Glied, geschwollen und spitz.

Halsschild $1 \frac{1}{2}$ mal so lang als breit, etwas schmäler als der Kopf, vorne gleichmäßig gerundet, an den Seiten hinter der Mitte mäßig eingeschnürt mit sehr kräftigem Seiteneindrucke. Basalrand deutlich, davor ein glatter, schmaler Streifen; Punktierung schwach und zerstreut, nur gegen die zweite Hälfte bis vor der glatten Stelle etwas dichter; ziemlich dicht, kurz, nicht ganz anliegend behaart.

Flügeldecken mehr als doppelt so lang als in der Mitte breit; Basis quer, Schultern etwas abfallend, mit spitzen Ecken; an der Spitze abgestuzt, Nahtecken abgestumpft; Nahtstreifen nur im letzten Drittel schwach sichtbar. Postskutellareindruck kaum wahrnehmbar.

Schildchen länglich-oval, in der Mitte flach. Äußerst schwach und zerstreut punktiert und behaart.

Füße kräftig; Tibien keulig. Vorderschenkel und Vordertibien des $\delta^{7}$ von ganz besonderer Gestalt, erstere an der Innenseite mit einem lappenförmigen Fortsatze, der in zwei Spitzen ausläuft (Fig. 4); Vordertibien in der Mitte stark erweitert und mit zwei spitz endenden Ausweitungen an der Innenseite.

Long. $5 \mathrm{~mm}$. (Ein on Hofmuseum in Wien.)

Borneo (ohne nähere Lokalität).

Zur Gruppe F. serdangus Mars., F. Bangi Pic etc. gehörend.

\section{Aulacoderus inopinans n. sp.}

Klein, oval, ganz schwarzbraun, (unausgefärbte Exemplare etwas lichter bräunlich, namentlich an der Spitze der Flügeldecken).

Kopf stark quer, mit breitbogiger in der Mitte mäßig einbuchteter Basis, Augen rund, grau, ziemlich vorstehend, Schläfen äußerst kurz. Fühler sehr schlank, kaum die Schultern erreichend; erstes und zweites Glied ziemlich dick, drittes bis achtes fadenförmig, allmälig kürzer werdend; neuntes und zehntes Glied breiter als die vorangehenden, gerundet, Endglied zirka dreimal so lang als das zehnte, sehr spitz. Ziemlich zerstreut, aber tief punktiert und mit wenigen kurzen, gelblichen Haaren bekleidet.

Halsschild quer, breiter als der Kopf, fast doppelt so breit als lang; die Seiten nach vorne mit ziemlich spitzer Ecke, gegen die Basis mäßig verengt, mit breitem, bogig geschweiftem Basalrande; ziemlich knapp davor ein mäßig tiefer, den ganzen Halsschild durchziehender und gleichfalls etwas bogig verlaufender Eindruck; der 
ganze Halsschild, insbesonders aber seine queren Seiteneindrücke mit gelblichen, kurzen Haaren bekleidet.

Flügeldecken an den Schultern nur wenig breiter als der Halsschild; Schultern stark nach aufwärts vorgezogen; Schulterecken deutlich, aber abgerundet; Schulterbeule breit; kein Postskutellareindruck; Omoplaten mäßig erhoben; Schildchen klein. Die Flügeldecken sind gegen die Mitte ziemlich stark erbreitet, konvex, gegen die Spitze allmälig verengt. Die Spitzen sind beim $\sigma^{\top}$ ausgeschweift und in eine Spitze vorgezogen; von dieser Spitze aus ist der Seitenrand eingebuchtet und mit einem eingestochenen Punkte versehen. Die Flügeldecken sind ziemlich zerstreut und seicht punktiert und mit gelblichen, nicht langen, borstenförmigen Haaren ziemlich dicht bekleidet.

Füße kurz, sehr schmächtig.

L 0 ng. $1 \cdot 8-2 \cdot 0 \mathrm{~mm}$.

Deutsch-Ost-Afrika, Vulkangebiet am Kiwu.

Diesen neuen Aulacoderus erwarb das Kgl. Museum in Berlin auf eine sonderbare Art. Major S c hl o b a ch hatte in Deutsch-Ost-Afrika zahlreiche, große Scarabaeiden, insbesondere Ateuchen, gesammelt und in eine Schachtel verpackt. Diese Tiere waren bei längerem Stehen in feuchter Gebirgsregion völlig eingefault und es hatte sich ein dicker Schimmel über sie gebreitet. In dieser Schachtel fanden sich nichts weniger als 286 Exemplare dieses neuen Aulacoderus. Das Merkwürdige ist aber dabei, daß sämtliche Exemplare o’ waren. Sie sind an der, auch bei anderen Spezies vorkommenden, besonderen Gestaltung der Flügeldecken leicht kenntlich. Nichtsdestoweniger schnitt ich 20 beschädigtere Exemplare auf und konnte bei allen den Penis präparieren. Der Penis ist durch einen mächtigen, mit einer dreifachen Reihe von Dornen bewehrten Präputialsack besonders ausgezeichnet.

Vielleicht können die folgenden, bei

\section{Formicomus Gestroi Pic}

gemachten Wahrnehmungen zur Klärung der Eigentümlichkeit, daß sich nur Männchen von Aulacoderus inopinans vorfanden, beitragen.

Ich erhielt unlängst seitens des Hamburger Museums eine größere Anzahl von Formicomus Gestroi aus Amani (Deutsch-OstAfrika), welche dort Dr. Eichelbaum in zwei Epochen gesammelt hatte, einmal am 4. Juli 1903 und später in der Zeit vom September bis November 1903. Von den in der ersten Epoche gesammelten 
Formicomus waren $20 \sigma^{\prime}$ und nur zwei $\$$, dagegen war das Verhältnis im Herbste umgekehrt, drei $\sigma^{\pi}$ und 20 을 Hienach kann wohl angenommen werden, dafo die Männchen dieser und wahrscheinlich auch anderer Arten früher ausschlüpfen, als die Weibchen; dafür lebt das o auch kürzere Zeit, da es bald nach vollzogener Begattung stirbt, während, gleichwie bei anderen Arten, das q noch längere Zeit leben muß, um für die Entwickelung der Nachkommenschaft Sorge zu tragen.

Es ist nicht unmöglich, daß die Schachtel mit Scarabaeiden gerade in einer Zeit Parasiten zugänglich war, da eben erst nur die $\sigma^{\top} \sigma^{\top}$ von Aulacoderus inopinans ausgeschlüpft waren. Andererseits ist aber auch eine parasitäre Metamorphose dieses Anthiciden nicht einfach von der Hand zu weisen, in welchem Falle man annehmen müßte, daß die $\sigma^{\prime}$ in der Schachtel selbst ausgeschlüpft seien, während für die $\bigcirc$ hiezu die Zeit noch nicht gekommen war, sie aber aus irgend einem Grunde

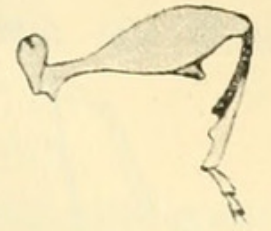

Fig. 5 . am späteren Ausschlüpfen verhindert waren.

Ich benütze diese Gelegenheit, um der Beschreibung des Herrn Maurice Pic (Ann. del Mus. di Genova 1894 p. 583 und Echange 1895 p. 19) über Formicomus Gestroi einiges auf Grund der Untersuchung zahlreicheren Materials hinzuzufügen: Das $\sigma^{\pi}$ hat an den vorderen Trochanteren einen aufrechten, aus breiter Basis spitz endenden Dorn, auch die mittleren Trochanteren weisen eine kleine, dornartige Erhebung auf (Fig. 5). An den Vordertibien des $\sigma^{\top}$ sind zwei dornartige Auswüchse wahrnehmbar, von welchen einer an der Unterseite mehr gegen das Tibienende, der andere an der Innenseite mehr in der Mitte der Tibie liegt.

Das $Q$ hat ebenfalls ein besonderes, charakteristisches Merkmal: das Mèsosternum ist nämlich in zwei Zapfen ziemlich stark vorgezogen.

Im übrigen ist Gestroi kein Anthelephilus, sondern ein Formicomus, u. zw. aus dem Grunde, weil er zum Fliegen geeignete Unterflügel hat. (Vgl. Lafertés Monographie, pag. 85.)

\section{Leptoprion nov. gen.}

Zwischen Hypaspistes und Notoxus stehend. Von ersterem durch das je vier Zähne an jeder Seite tragende Horn, durch die kürzeren, nur bis zur Körpermitte reichenden Fühler, durch die flacheren, stärker behaarten Flügeldecken verschieden; auch sind bei Leptoprion die Tarsen etwas kürzer als die Tibien. Von Notoxus 
unterscheidet sich das neue Genus vornehmlich durch das viel längere, schmälere und gröber gezähnte Horn und durch die längeren Füße.

\section{Leptoprion angulatus n. sp. (Fig. 6).}

Ziemlich lang mit breiten, an den Schultern spitzwinkeligen Flügeldecken. Kopf, Halsschild und Horn dunkelrotbraun, letzteres an der Spitze etwas heller. Fühler und

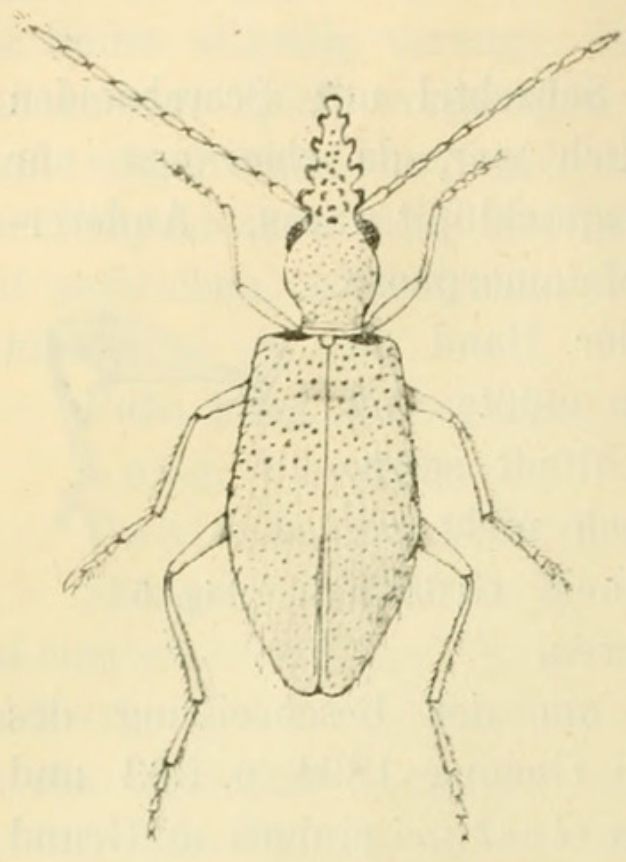

Fig. 6.

Leptoprion angulatus $\mathrm{n}$. $\mathrm{sp}$. Mundteile rotbraun, die ersten zwei Fühlerglieder lichter; Flügeldecken ganz dunkelbraun, nur die Naht etwas rötlich; Füße blaßgelb.

Kopf länger als breit, vorn gerade, mit deutlichem, unregelmäßigem Stirneindrucke. Augen sehr groß, eirund, grob facettiert, mit feinen, anliegenden, nach vorne gerichteten, kurzen Haaren; die Oberlippe mit stärkeren, dichteren und längeren Haaren bekleidet. Schläfen äußerst kurz. Fühler gegen die Spitze fast gar nicht verdickt, die Hälfte des Körpers erreichend, dicht, gelblich behaart; erstes Glied sehr lang, nach vorne ziemlich verdickt, zweites Glied kurz, die folgenden Glieder gleichmäßig länger, nur das Endglied etwas länger als das zehnte Glied, spitz. Letztes Palpenglied lang gestreckt, etwas eiförmig.

Horn an der Wurzel ein wenig erhoben und dann leicht nach vorne geneigt. Jeder Zahn mit einer erhobenen Verdickung am Rande. Zwischen den Zähnen ist der mäßig erhobene, runzelige Kamm mit 18 unregelmäßigen, dunkelroten, zum Teil halbkreisförmigen, wulstigen, zum Teil knopfartigen Erhebungen versehen. Der Halsschild ist grob und zerstreut punktiert; das Horn oben und unten mit ziemlich groben, gekrümmten Haaren, das Halsschild mit längeren, zum Teile quergestellten, gelblichen Haaren ziemlich dicht bekleidet; überdies einzelne lange, abstehende Haare. Halsschild länglich, gerundet, von der Breite des Kopfes, an den Seiten, ziemlich entfernt von der Basis, leicht eingeschnürt, die Seiten, an der Einschnürung gerade zum schmalen Basalrande gerichtet.

Flügeldecken mehr als doppelt so breit als der Halsschild in seiner größten Breite, doppelt so lang als breit; Schultern sehr eckig; 
Seiten bis zur Mitte breiter, dann bis zur einzeln, etwas schräg abgerundeten Spitze wieder schmäler werdend; eher flach, mit sehr seichtem Postskutellareindrucke; sehr glänzend, mit zerstreuten, groben Punkten, woraus gelbliche, halb aufrechte, längliche Haare entspringen. Schildchen länglich dreieckig, mit tiefem Eindrucke. Nahtstreifen schmal, in der zweiten Hälfte schwach erhoben.

Füße lang, sehr schlank, Schenkel kaum verdickt, ganz blaßgelb, mit Borsten und Dornen dicht bekleidet. Klauenglied der Vorder- und Mittelfüße stark ausgebuchtet. Hintertibien etwas sichelförmig.

L o ng. $\quad 3 \cdot 2 \mathrm{~mm}$.

Sum atra: Manna. Ein Exemplar (ohne deutliche äußere Sexualcharaktere) in der Sammlung des Herrn Dr. H. J. Veth am Haag.

\section{Pseudoleptaleus arcuatus n. sp.}

Klein, sehr glänzend. Halsschild, Schenkel, Tarsen, die ersten Fühlerglieder und Kopf (letzterer dunkler) mehr minder gelbrot. Auch die Tibien von dieser Farbe, aber namentlich jene der Vorderfüße in der Mitte gedunkelt. Flügeldecken dunkel rotbraun, das Basisdrittel gelb, um das Schildchen rötlicher braun, manchmal auch die Schulterecke kurz gebräunt.

Kopf oval, convex, Augen klein, quer, rund, etwas hervorstehend. Schläfen ziemlich lang, in gleichmäßiger Rundung zur runden Basis übergehend. Nicht dicht, unregelmäßig, ziemlich tief punktiert; Behaarung äußerst schwach. Fühler über die Schultern reichend, schlank, gegen die Spitze mäßig verdickt; erstes Glied länger und dicker, zweites Glied kürzer als die folgenden; achtes, neuntes und zehntes Glied allmälig kugelig werdend, Endglied

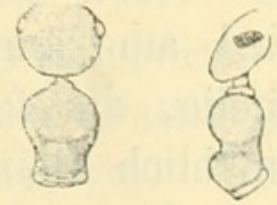

Fig. 7. Fig. 8 . $1 \frac{1}{2}$ mal so lang als das vorangehende, spitz; schwach behaart. Letztes Palpenglied etwas beilförmig, spitz, gerandet.

Halsschild etwas schmäler als der Kopf, länger als breit, Vorderlobus zwei Drittel der Halsschildlänge einnehmend, gleichmäßig gerundet; Einschnürung stark und ziemlich tief, namentlich an den Seiten; Hinterlobus mit buckelig erhöhter Mitte. Basalrand ziemlich breit. Punktierung äußerst schwach und zerstreut. Mit dünnen gelblichen, anliegenden Haaren schwach bekleidet. (Fig. 7 und 8.) Flügeldecken an den Schultern doppelt so breit als der Halsschild, in der Mitte ziemlich stark erbreitert, dann verschmälert zur gemeinsam gerundeten Spitze; kaum doppelt so lang, als in der Mitte breit; sehr schwach und zerstreut punktiert, aus jedem Punkte ein schwaches 
Haar entspringend, Schultern gerade mit spitzer Ecke; Omoplaten erhoben; Postskutellareindruck deutlich und namentlich an der Naht tief; Schildchen klein, spitz. Seitenrand der Flügeldecken überall schmal erhoben.

Füße ziemlich lang, Schenkel nicht verdickt, Tibien relativ kräftig, namentlich die Hintertibien etwas sichelförmig und ziemlich erbreitert; $\sigma^{\prime}$ mit vorstehendem Pygidium; letztes Sternit in der Mitte gegen den Rand mit rundlichem Eindrucke.

L o ng. $2 \cdot 2 \mathrm{~mm}$.

Sumatra: Manna. Dem Pseudoleptaleus trigibber Mars. aus Japan und China nahestehend, aber durch den anders gebildeten Halsschild verschieden.

(Coll. Dr. Veth am Haag und Hofmuseum Wien).

\section{Tomoderus borneensis n. sp.}

Groß, mit sehr erbreiterten und konvexen Flügeldecken, ganz braunrot, glänzend, die Tarsen und die zwei Endglieder der Fühler etwas lichter.

Kopf stark quer, viel breiter als lang; Augen klein, rund etwas vorstehend; sehr zerstreut und fein punktiert, mit wenigen, schwachen, anliegenden und einzelnen abstehenden Haaren bekleidet. Fühler die Schultern erreichend, von ungewöhnlicher Gestalt: erstes Glied schmäler als die Mittelglieder, zweites Glied sehr klein und, im Verhältnisse zu den folgenden Gliedern, sehr schmal; drittes Glied am längsten von allen anderen, viertes und fünftes etwas kugelig, die folgenden stark quer, das fünfte am breitesten, dann allmählich kleiner werdend, Endglied $1 \frac{1}{2}$ mal so lang als das zehnte Glied. Ziemlich dicht behaart. - Zwischen den Fühlerwurzeln kein Quereindruck.

Halsschild viel schmäler als der Kopf, $1 \frac{3}{4}$ mal länger als breit, mit breiter Einschnürung. Diese ist dunkler, dicht runzelig und weist an ihren Seiten unten eine wulstartig erhöhte, dunkle Linie auf. Sonst ist der Halsschild sehr zerstreut, unregelmäßig und fein punktiert und mit Ausnahme einiger abstehender Haare kaum behaart. Der Hinterlobus ist an der Basis so breit, wie der Vorderlobus in der Mitte; die Basis ist fein gerandet, der Rand gegen die Seiten hin breiter.

Flügeldecken an der Basis mehr als doppelt so breit als der Halsschild, doppelt so lang als hinter der Mitte breit; an den Seiten stark erbreitert, hinter der Mitte am breitesten, die Seitenränder die Unterseite stark umfassend, dann quer abgestutzt zur 
Spitze; Schultern vorgezogen, mit gerundeten Ecken; Schildchen herzförmig; Nahtstreifen sehr schmal. Globos, ziemlich stark, aber zerstreut und verworren punktiert (die Stärke der Punkte gegen die Spitze abnehmend), mit halbaufrechten nicht langen, gelblichen, dazwischen auch mit mehr vereinzelten, längeren, ganz aufrechten Haaren ziemlich dicht bekleidet.

Füße kräftig, alle Schenkel keulenförmig.

L ong. $3.4 \mathrm{~mm}$.

Süd-B or n e 0: Pengaron (ein Exemplar im Hofmuseum in Wien).

An Gestalt und Größe dem Tomoderus clavipes Champ. aus Japan ähnlich, aber durch den schmäleren, kräftiger eingeschnürten Halsschild, durch die besondere Gestalt der Fühler, sowie auch durch die zerstreutere und gröbere Punktierung der Flügeldecken verschieden.

\section{Anthicus-Arten mit erweiterten Fühlergliedern.}

Von den Anthicus-Arten, welche einzelne, lappenförmig erweiterte Fühlerglieder aufweisen, sind beschrieben:

Anthicus diversicornis Pic (Ann. Soc. Ent. Fr. 1907, p. 195), aus Kashmir und $\mathrm{Kulu}$, und

Anthicus monstrosicornis Mars. (Ann. Soc. Ent. Fr. 1876, p. 465) aus Indien.

Ersterer hat das vierte, fünfte und sechste Fühlerglied nach der Unterseite lappenförmig erweitert; bei letzterem trifft dies nur hinsichtlich des fünften und sechsten Fühlergliedes zu, wobei das sechste Fühlerglied etwas mehr ausgezogen ist als das vorangehende.

Anthicus monstrosicornis kommt auch auf Ceylon (Nalanda), dann auf Borneo (Südosten der Insel) und in Japan vor. Die Ceyloner Exemplare weisen fast durchgehends eine viel breitere, dunkelbraune Medianmakel auf als die Japaner und Borneo-Exemplare. Letztere sind etwas weniger grob, aber dichter punktiert, auch dichter und länger behaart, als die indischen und japanischen Stücke.

Ich besitze einen Anthicus aus dem Nimrod-Sund (Provinz Tschekiang in Ostchina), dessen fünftes und sechstes Fühlerglied ebenfalls, wenn auch nicht so stark, wie bei Anthicus monstrosicornis, lappenförmig erweitert ist, der aber im übrigen von diesem spezifisch verschieden ist, und in mancherlei Beziehung an die LeptaleusArten erinnert:

Anthicus aemulus n. sp. Schlank, ganz lichtrotbraun, nur der Kopf dünkler; die Flügeldecken in der zweiten Hälfte gedunkelt, die dunkle Farbe gegen die Spitzen der Flügeldecken abnehmend, Beine blaßgelb. 
Kopf langgestreckt, mehr als $1 \frac{1}{2}$ mal länger als breit, hinten gerundet, am Scheitel ganz glatt, gegen die Augen grob, tief, zerstreut und unregelmäßig punktiert. Augen rund, sehr nach vorne gerückt, wenig vortretend, Schläfen lang, parallel. Fühler von der Hälfte der Körperlänge, gegen die Spitze mäßig verdickt, Endglied $1 \frac{1}{2}$ mal so lang als das zehnte Glied, länglich eirund, kaum gespitzt; fünftes und sechstes Glied mäßig lappenförmig erweitert.

Halsschild wie bei monstrosicornis, sehr zerstreut, grob punktiert, viel breiter als der Kopf.

Flügeldecken mehr als doppelt so lang als breit, an den Seiten fast parallel, Schultern vorgezogen, eckig mit deutlicher Schulterbeule, Spitzen abgestutzt, Nahtecken etwas gerundet; sehr grob, nicht dicht, in Reihen punktiert; die Pnnktierung gegen die Spitze allmählich abnehmend; mit halbaufrechten gelben, ziemlich langen Haaren nicht dicht bekleidet. Nahtstreifen in der zweiten Hälfte mäßig erhoben. Pygidium dunkel. Füße lang, schlank, Schenkel mäßig verdickt, hellgelb, Wurzeln der Tibien etwas gerötet. Hintertarsen fast so lang als die Tibien. Hintertibien, im Gegensatze zu monstrosicornis ohne Ausbuchtung beim $\sigma^{\pi}$.

Long. $24 \mathrm{~mm}$. (Ein $\sigma^{\top}$.) Sammlung Krekich.

Eine weitere Art mit eigentümlicher Fühlerbildung beim $\sigma^{\lambda}$ ist Anthicus brevisignatus Pic (Echange 1901, p. 33, 98) aus Sïdafrika. Das sechste Fühlerglied ist bei dieser Art nach unten mehr oder minder spitz, lappenförmig erweitert.

Anthicus brevisignatus ist in der Sammlung des British Museum in zahreichen typischen Stücken enthalten. Auch das Hofmuseum in Wien besitzt einige Exemplare. Er ist in der Färbung außerordentlich veränderlich, wie aus nachstehender Zusammenstellung der mir vorliegenden Exemplare hervorgeht:

Aus Dumbrody (Juni 1900): Kopf und Flügeldecken ganz dunkelbraun; Halschild braunrot mit gedunkelten Vorderecken; die vier Makeln der Fügeldecken gelbrot; Füße hellgelbbraun, mit gedunkelten Schenkelspitzen.

Aus Dumbrody (November 1903): Kopf und Flügeldecken dunkelbraun; die vier Makeln der Flügeldecken orangegelb; Fühler und Füße ganz lichtgelb; Halsschild hellgelb.

Vom Tafelberge: Ganz dunkelbraun, fast schwarz, nur der Basalrand des Halsschildes rotbraun; die vier Makeln der Flügeldecken orangegelb, die Fühler dunkelbraun, nur die Basalglieder etwas heller; die Schenkel dunkelbraun mit hellerer Wurzel; Tibien und Tarsen orangegelb. 
Aus Salisbury (Mashonaland, November 1899). Wie die Exemplare vom Tafelberge, aber lichtere Mittelschenkel, Halschild rotbraun, Fühler orangegelb, mit gedunkelten Mittelgliedern.

Aus Salisbury (gleiches Datum). Ähnlich dem vorigen, aber die Fühler und Füße ganz gelb, der Halsschild rotgelb, mit gedunkelten Vorderecken, Kopf und Flügeldecken aber sehr dunkel, fast schwarz.

Aus Transval. Kopf und Flügeldecken ganz dunkelbraun. Fühler in der Nitte gedunkelt, Füße braungelb, mit gedunkelter Schenkelkeule; Halsschild und die vier Makeln der Flügeldecken rötlichbraun.

Aus Natal (März 1896). Ganz helle Färbung (wahrscheinlich unreifes Exemplar).

Angesichts dieser Veränderlichkeit glaube ich, daß die von M. Pic aufgestellte var. subsignatus für die dunkleren Exemplare nicht berechtigt ist. Aber es ist auch fast gewiß, daf A. brevisignatus mit A. quadrillum Laf. identisch ist. Weder La Ferté noch Pic hat die besondere Form der Fühler des o hervorgehoben. Die Beschreibung des $A$. quadrillum paßt aber vollkommen auf den typischen brevisignatus. Wenngleich La F erté von der Ähnlichkeit der Zeichnung der Flügeldecken des quadrillum mit jener des Leptaleus Klugi spricht, so deutet doch die Benennung "quadrillum" darauf hin, daß dem Autor Exemplare mit vier Makeln auf den Flügeldecken vorlagen ( $L$. Klugi hat gewöhnlich vorne eine Basalbinde und gegen die Spitze zwei Makeln).

Der Umstand, daf A. brevisignatus in Südafrika weit verbreitet ist, erhöht die Wahrscheinlichkeit, daß er mit A. quadrillum identisch ist.

Anthicus quadrillum Laf ( $=$ A. brevisignatus Pic et var. subsignatus Pic) gehört bis auf weiteres vermöge der Gestaltung seines Halsschildes in die unmittelbare Nähe der Gruppe V (Cyclodinus) Marseul's und zwar zu jenen wenigen Arten, die - wie Anthicus erro Truq. - keine Tuberkeln vor der Basis des Halsschildes aufweisen.

Auf Madagaskar kommt weiters ein Anthicus vor, der mit A. quadrillum die größte Ähnlichkeit hat und dessen ơ gleichfalls das sechste Fühlerglied stark und zwar fast rechtwinkelig ausgezogen hat.

Dieser Anthicus, welchen ich als eine Variätet - v. nov. consanguineus - des Anthicus quadrillum ansehe, unterscheidet sich von letzterem wie folgt: Etwas größer, Kopf und Flügeldecken gewölbter, Augen viel weniger vortretend, Endglied der Fühler spitziger, 
der lappenförmige Fortsatz des sechsten Fühlergliedes rechtwinkelig (bei $A$. quadrillum schiefwinkelig), Flügeldecken zerstreuter und feiner punktiert, Schenkel kräftiger; endlich befindet sich die Ausbuchtung der Hintertibien des $\sigma^{\top}$ bei $A$. quadrillum ungefähr in der Mitte, dagegen bei der v. consanguineus mehr gegen die Wurzel und ist die ganze Tibie des letzteren mehr geschwungen. -

$\mathrm{Zu}$ den Anthicus-Arten mit erweiterten Fühlergliedern sind schließlich noch zu zählen:

Anthicus Marseuli Pic $=$ scoticus Mars. aus Japan (Ann. Soc. Ent. Fr. 1876 , p. 462 und $1892-1893$, p. CCXI) mit stark lappenförmig ausgezogenem vierten und fünften Fühlergliede, dann die mit diesem verwandten

Anthicus cohaeres Lewis (Ann. Mag. Nat. Hist. 1895, p. 430) und Anthicus extus Lewis (Ibid), beide gleichfalls aus Japan, die dieselbe Gestaltung der Fühler, wenn auch in schwächerem Maasse aufweisen; endlich

Anthicus formosanus Pic aus Formosa (Mél. exot. entom. Fasc. I., p. 11, Arch. f. Naturgesch. 1913, p. 133), bei welchem die Fühlerglieder $3-6$ verdickt und ausgezogen sind. 


\section{$2 \mathrm{BHL}$ Biodiversity Heritage Library}

Krekich-Strassoldo von Treuland, Hans. 1914. "Neue Anthiciden und Mitteilungen über die Verbreitung bekannter Anthiciden." Wiener entomologische Zeitung 33, 1-14. https://doi.org/10.5962/bhl.part.17773.

View This Item Online: https://www.biodiversitylibrary.org/item/42670

DOI: https://doi.org/10.5962/bhl.part.17773

Permalink: https://www.biodiversitylibrary.org/partpdf/17773

\section{Holding Institution}

Smithsonian Libraries

\section{Sponsored by}

Smithsonian

\section{Copyright \& Reuse}

Copyright Status: NOT_IN_COPYRIGHT

This document was created from content at the Biodiversity Heritage Library, the world's largest open access digital library for biodiversity literature and archives. Visit BHL at https://www.biodiversitylibrary.org. 